\title{
Thermal Instability of Self-Gravitating Rotating Viscous Partially Ionized Plasma under the Effect of Hall Current
}

\author{
A.K. Patidar ${ }^{1}$, V. Shrivastava ${ }^{2}$, R. K. Pensia ${ }^{3}$ \\ 1,2 Department of Physics, Pacific University, Udaipur, 313024 (Raj.) India \\ ${ }^{3}$ Department of Physics, Govt. Girls College, Neemuch, 458441 (M. P.) India
}

\begin{abstract}
The problem of thermal instability of an infinitely extending homogeneous self-gravitating partiallyionized gaseous plasma, which is permeated by uniform magnetic field, has been investigated in this paper in the presence of radiative heat-loss functions, rotation, viscosity, thermal conductivity, electrical resistivity and Hall current of the medium. With the help of relevant linearized perturbation equations of the problem, a general dispersion relation is obtained for the considered medium and discussed in the longitudinal and transverse direction of propagation by using normal mode analysis method. The longitudinal mode is found to be modified by Alfven speed, rotation, viscosity and Hall current of the medium and it is also found that the condition of radiative instability is independent of the magnetic field, Hall current and viscosity but for the transverse mode of propagation it depends on the strength of the magnetic field. The damping effect is produced due to collision frequency, viscosity and Hall current. The curve shows the stabilizing effects of neutral component, rotation and magnetic field and destabilizing effect of thermal conductivity, electrical resistivity and density-dependent heat-loss functions on the growth rate of instability of the system.
\end{abstract}

Keywords: Jeans Instability; Thermal Conductivity; Radiative Heat-Loss Function; Interstellar Medium (ISM); Collision-Frequency.

\section{Introduction}

There has been a great deal of interest in studying various collective processes in gaseous plasma, which are ubiquitous in space, including diffuse and dense interstellar media, star envelops, accretion disks, circumstellar shells, chromosphere, dark interiors and the out flow of red giant star. Thus, in to the crucial phenomena of the interstellar medium (ISM), many body gravitating system play an essential role. The gravitational instability is of the fundamental concept of modern astrophysical plasma and it is connected with the fragmentation of interstellar matter in regard to star formations. James Jeans [1] first studied this instability problem and shows that an infinite homogeneous, self-gravitating fluid is unstable for all wave number which is less then critical Jeans wave number. Chandrashekhar [2] has given the comprehensive account of the effect of a magnetic field and rotation separately and simultaneously on the gravitational instability of an infinite homogeneous medium and observed that the Jeans criterion remains unaffected in each case. The Jeans instability has been extensively investigated under varying assumption.

Along with this it is an established fact that magnetic fields play an important role in interstellar gas dynamics. In the interstellar medium (ISM), a large amount of energy is injected by stars, which leads to the formation of shock waves; but when these shocks waves weaken, they become large amplitude hydromagnetic Alfven waves. Langer [3] studied the importance of magnetic field in the stability of ISM molecular clouds and he suggested that the magnetic fields can provide pressure support and inhibit the contraction and fragmentation of interstellar clouds. The magnetic field interact directly only with the ions, electrons and charged grains in the gas. Collision of the ions with the predominantly neutral gas in the clouds is responsible for the incident coupling of the magnetic field to the bulk of the gas. The degree to which the magnetic pressure is important depends upon the field's strength and the fractional abundance. In addition ion would undergo contraction in one dimension until the density increases to a value sufficient to cause collapse the field lines. In this connection, many investigators have discussed the gravitational instability of a homogeneous plasma considering the effect of magnetic field incorporating various parameters. \{Mestel and Spitzer [4], Mestel [5], Spitzer $[6,7]\}$.

In this way, the effect of Hall current on plasma stability has been extensively examined \{Ariel [8], Bhowmick [9], Ali and Bhatia [10]\} leading to the conclusion that the Hall currents are destabilizing in nature. Many researcher, like Kumar [11], Chhajlani and Vaghela [12] centered their studies on thermally conducting plasma under varying assumptions. Vyas and Chhajlani [13], Sharma and Chand [14], Khan and Bhatia [15] have investigated the influence of permeability of porous medium on plasma instability in view of the importance of such studied in geology and heavy oil recovery. Bhatia and Chhonkar [16] have studied the combined influence of Coriolis force and viscosity on plasma stability in the presence of Hall currents and concluded that viscosity has a stabilizing influence on the system. In all such investigations, carried out 
separately under varying assumption, it was found that the condition of instability has been determined by Jeans criterion with some modifications.

Recently, Lima et al. [17] have studied the problem of Jeans gravitational instability and non-extensive kinetic theory. Khan and Shaikh [18] have discussed the instability of thermally conducting self-gravitating system. The role of magnetic field in contraction and fragmentation of interstellar clouds has been studied by Pensia et al. [19]. Thus we find that the problem of Jeans gravitational instability is the important phenomena to understand gravitational collapse of the Protostar. Stiele et al. [20] have discussed clump formation due to thermal instability in weakly ionized plasma. Inutsuka et al. [21] have investigated the propagation of shock waves into a warm neutral medium taking into account radiative terms. Menou et al. [22] have shown the importance of radiative effects in the sun's upper radiative zone. Kim and Narayan [23] have discussed the thermal instability in clusters of galaxies with conduction taking on the role of the effect of radiative heat-loss function. Shadmeri and Dib [24] have discussed the thermal instability in a magnetized partially ionized plasma with charged dust particles and radiative cooling functions. Fukue and Kamaya [25] have explored the problem of thermal instability considering the effects of ion-neutral friction, radiative cooling functions and magnetic field. Shaikh et al. [26, 27] have explored the problem of gravitational instability of thermally conducting partially ionized plasma in a variable magnetic field, considering the effects of Hall currents, finite conductivity, ion viscosity, thermal conductivity and collision with neutrals.

From the above studies, we find that thermal conductivity, interaction between two components of partially ionized plasma and radiative heat-loss functions are the important parameters to discuss the problem of gravitational instability. Thus in present problem, we investigate the effects of radiative heat-loss functions, rotation, viscosity, Hall current, thermal conductivity and electrical resistivity on the Jeans instability of partially ionized plasma

\section{Equations of the Problem}

Let us consider of a homogeneous composite partially ionized plasma including Hall current, radiative heat-loss functions, viscosity, finite electrical resistivity and thermal conductivity, rotation under the influence of uniform magnetic field $\vec{H}(0,0, H)$. We assume that the two components of the partially ionized plasma (the ionized fluid and the neutral gas) behave like a continuum fluid and their state velocities are equal etc. are considered, have the following form.

$$
\begin{aligned}
& \frac{d \overrightarrow{\mathrm{v}}}{d t}-2(\overrightarrow{\mathrm{v}} \times \vec{\Omega})=-\frac{\vec{\nabla} p}{\rho}+\vec{\nabla} \phi+\frac{1}{4 \pi \rho}(\vec{\nabla} \times \vec{H}) \times \vec{H}+\frac{\rho_{d}}{\rho} v_{c}\left(\overrightarrow{\mathrm{v}}_{d}-\overrightarrow{\mathrm{v}}\right)+v \nabla^{2} \overrightarrow{\mathrm{v}} . \\
& \frac{d \overrightarrow{\mathrm{v}}_{d}}{d t}=-v_{c}\left(\overrightarrow{\mathrm{v}_{\mathrm{d}}}-\overrightarrow{\mathrm{v}}\right) . \\
& \frac{d \rho}{d t}=-\rho \vec{\nabla} \overrightarrow{\mathrm{v}} . \\
& \nabla^{2} \phi=-4 \pi G \rho . \\
& \frac{1}{(\gamma-1)} \frac{d p}{d t}-\frac{\gamma}{(\gamma-1)} \frac{p}{\rho} \frac{d \rho}{d t}+\rho \mathcal{L}-\vec{\nabla} \cdot(\lambda \vec{\nabla} T)=0 . \\
& p-\frac{R}{\mu} \rho T=0 . \\
& \frac{d \vec{H}}{d t}=\vec{\nabla} \times(\overrightarrow{\mathrm{v}} \times \vec{H})+\eta \nabla^{2} \vec{H}-\frac{c}{4 \pi N e}[\vec{\nabla} \times\{(\vec{\nabla} \times \vec{h}) \times \vec{H}\}] . \\
& \vec{\nabla} \cdot \vec{H}=0 .
\end{aligned}
$$

where $\vec{\Omega}\left(\Omega_{\mathrm{x}}, 0, \Omega_{\mathrm{z}}\right), \mathrm{v}_{d}, p, \gamma, \phi, \vec{h}\left(h_{x}, h_{y}, h_{z}\right), \mathrm{G}, \lambda, \mathrm{R}, c, N, \rho, \rho_{d} T, \eta, v, e$, and $\overrightarrow{\mathrm{v}}\left(\mathrm{v}_{\mathrm{x}}, \mathrm{v}_{\mathrm{y}}, \mathrm{v}_{\mathrm{z}}\right)$ denote the rotational frequency, neutral gas velocity, pressure, ratio of specific heats, gravitational potential, magnetic field, gravitational constant, coefficient of thermal conductivity, gas constant, velocity of light, number density, density of ionized component, density of neutral components $\left(\rho \gg \rho_{d}\right)$, temperature, finite electrical resistivity, kinematic viscosity, charge of electron, and the gas velocity, respectively. Operator $d / d t$ is the substantial derivative given by

$$
\frac{d}{d t}=\left(\frac{\partial}{\partial t}+\overrightarrow{\mathrm{V}} \cdot \vec{\nabla}\right)
$$

\section{Linearized Perturbation Equations}

The space and time dependent physical quantities $\overrightarrow{\mathrm{v}}, p, \rho, T, \vec{H}$ and $\phi$ can be written as the sum of the equilibrium and perturbed part

$\rho=\rho_{0}+\delta \rho, \quad p=p_{0}+\delta p, \quad \vec{H}=\vec{H}_{0}+\delta h, \quad T=T_{0}+\delta \mathrm{T}, \quad \phi=\phi_{0}+\delta \phi, \quad \overrightarrow{\mathrm{v}}=\overrightarrow{\mathrm{v}}_{0}+\delta \overrightarrow{\mathrm{v}},\left(\right.$ with $\left.\mathrm{v}_{0}=0\right)$, $\mathcal{L}=\mathcal{L}_{0}+\delta \mathcal{L},\left(\right.$ with $\mathcal{L}_{0}=0$ )

The term with subscript ' 0 ' denotes the equilibrium part of the physical quantities. Using equation (I)(II) in equation (1)-(8), we can write the linearized perturbation equation of finite electrical conducting, rotating, viscous, partially ionized plasma with radiative effect, magnetic field and thermal conductivity, removing ' 0 ' from subscripts in the equilibrium quantities for simplicity. Thus we obtain 


$$
\begin{aligned}
& \frac{\partial \overrightarrow{\mathrm{v}}}{\partial t}-2(\overrightarrow{\mathrm{v}} \times \Omega)=-\vec{\nabla} \frac{\delta p}{\rho}+\vec{\nabla} \delta \phi+\frac{1}{4 \pi \rho}(\vec{\nabla} \times \vec{h}) \times \vec{H}+\frac{\rho_{d}}{\rho} v_{c}\left(\overrightarrow{\mathrm{v}}_{d}-\overrightarrow{\mathrm{v}}\right)+\nu \nabla^{2} \overrightarrow{\mathrm{v}} . \\
& \frac{\partial \overrightarrow{\mathrm{v}}_{d}}{\partial t}=-v_{c}\left(\overrightarrow{\mathrm{v}_{\mathrm{d}}}-\overrightarrow{\mathrm{v}}\right) . \\
& \frac{\partial \delta \rho}{\partial t}=-\rho \vec{\nabla} \overrightarrow{\mathrm{v}} . \\
& \nabla^{2} \delta \phi=-4 \pi G \delta \rho . \\
& \frac{1}{(\gamma-1)} \frac{\partial \delta \rho}{\partial t}-\frac{\gamma}{(\gamma-1)} \frac{p}{\rho} \frac{\partial \delta \rho}{\partial t}+\rho\left(\mathcal{L}_{\rho} \delta \rho+\mathcal{L}_{\mathrm{T}} \delta \mathrm{T}\right)-\lambda \nabla^{2} \delta \mathrm{T}=0 . \\
& \frac{\delta p}{p}=\frac{\delta T}{T}+\frac{\delta \rho}{\rho} . \\
& \frac{\partial \vec{h}}{\partial t}=\vec{\nabla} \times(\overrightarrow{\mathrm{v}} \times \vec{H})+\eta \nabla^{2} \vec{h}-\frac{c}{4 \pi N e}[\vec{\nabla} \times\{(\vec{\nabla} \times \vec{h}) \times \vec{H}\}] . \\
& \vec{\nabla} \cdot \vec{h}=0 .
\end{aligned}
$$

Here $\mathcal{L}_{\rho, T}$ are the partial derivatives of the density dependent $(\partial \mathcal{L} / \partial \rho)_{T}$ and temperature dependent $(\partial \mathcal{L} / \partial T)_{\rho}$ heat-loss functions respectively.

\section{Dispersion Relation}

In order to study the stability of the system we assume that all the perturbed quantities vary as $\exp \left\{i\left(k_{x} x+k_{z} z+\omega t\right)\right\}$.

where $k_{x(z)}$ represents the wave number in the perpendicular $\mathrm{x}$, and parallel $\mathrm{z}$ direction to the magnetic field such that $k_{x}^{2}+k_{z}^{2}=k^{2}$. The harmonic frequency of the perturbation is denoted by $\omega$. Combining equation (13) and (14), we obtain the expression for $\delta p$ as.

$$
\begin{aligned}
& \begin{aligned}
\delta p & \{\sigma+\beta\}=\left\{\alpha+\sigma C^{2}\right\} \delta \rho . \\
\text { where } \sigma=i \omega, C=\left(\frac{\gamma p}{\rho}\right)^{1 / 2} & \text { is the adiabatic velocity of soun } \\
\alpha & =(\gamma-1)\left(\mathcal{L}_{T} T-\mathcal{L}_{\rho} \rho+\frac{\lambda k^{2} T}{\rho}\right) . \\
\beta & =(\gamma-1)\left(\frac{\mathcal{L}_{T} T \rho}{p}+\frac{\lambda k^{2} T}{p}\right) .
\end{aligned}
\end{aligned}
$$
are given by

Using equation (10)-(18) in equation (9), we obtain the following algebraic equations for the amplitude components

$$
\begin{aligned}
& \left(R_{1}+\frac{k^{2} V^{2} d}{A}\right) \mathrm{v}_{x}-\left(\frac{k_{z}^{2} Q V^{2} k^{2}}{A}+2 \Omega_{\mathrm{z}}\right) \mathrm{v}_{y}+\frac{i k_{x}}{k^{2}} \Omega_{\mathrm{T}}^{2} \mathrm{~s}=0 . \\
& \left(\frac{k_{z}^{2} Q V^{2} k^{2}}{A}+2 \Omega_{\mathrm{z}}\right) \mathrm{v}_{x}+\left(R_{1}+\frac{k_{Z}^{2} V^{2} d}{A}\right) \mathrm{v}_{y}-2 \Omega_{\mathrm{x}} \mathrm{v}_{z}=0 . \\
& 2 \Omega_{\mathrm{x}} \mathrm{v}_{y}+\mathrm{R}_{1} \mathrm{v}_{z}+\frac{i k_{z}}{k^{2}} \Omega_{\mathrm{T}}^{2} \mathrm{~S}=0 .
\end{aligned}
$$

Taking divergence of equation (9) with addition of equation (10)-(18), we obtain

$$
\left(\frac{i k_{x} k^{2} V^{2} d}{A}\right) \mathrm{v}_{x}-\left(i k_{x} \frac{k_{Z}^{2} Q V^{2} k^{2}}{A}+2 i k_{x} \Omega_{\mathrm{z}}-2 i k_{z} \Omega_{\mathrm{x}}\right) \mathrm{v}_{\mathrm{y}}-\left(\sigma R_{1}+\Omega_{\mathrm{T}}^{2}\right) s=0 .
$$

where $s=\frac{\delta \rho}{\rho}$ is the condensation of the medium, $V=\frac{H}{(4 \pi \rho)^{1 / 2}}$ is the Alfven velocity, $C^{2}=\gamma C^{2}$ where $C$ and $C^{\prime}$ are the adiabatic and isothermal velocities of sound. Also we have assumed the following substitutions

$$
\begin{aligned}
& d=\sigma+\Omega_{m}, \Omega_{m}=\eta k^{2}, \Omega_{\mathrm{v}}=v k^{2}, R_{1}=\sigma+\frac{\sigma B v_{c}}{\sigma+v_{c}}+\Omega_{\mathrm{v}}, \Omega_{\mathrm{I}}^{2}=k^{2} \alpha-4 \pi G \rho \beta, \\
& \Omega_{j}^{2}=k^{2} C^{2}-4 \pi G \rho, \Omega_{T}^{2}=\frac{\sigma \Omega_{j}^{2}+\Omega_{I}^{2}}{\sigma+\beta}, Q_{1}=\frac{k_{z}^{2} Q V^{2} k^{2}}{A}, Q=\frac{c H}{4 \pi N e}, A=d^{2}+k_{z}^{2} Q^{2} k^{2},
\end{aligned}
$$

Equation (19)-(22) can be written as

$$
[\mathrm{X}][\mathrm{Y}]=0 .
$$

where $[X]$ is the fourth order square matrix and $[Y]$ is a single column matrix whose elements are $v_{x}, v_{y}$, $\mathrm{v}_{\mathrm{z}}$ and $s$. For a non- trivial solution of the equation (23) the determinant of the matrix [X] should vanish, leading to the general dispersion relation.

$$
\begin{aligned}
& {\left[-\left\{\left(R_{1}+\frac{k^{2} V^{2} d}{A}\right)\left(R_{1}+\frac{k_{z}^{2} V^{2} d}{A}\right)\left(\sigma R_{1}+\Omega_{\mathrm{T}}^{2}\right) R_{1}\right\}-4\left(R_{1}+\frac{k^{2} V^{2} d}{A}\right)\left(\sigma R_{1}+\Omega_{\mathrm{T}}^{2}\right) \Omega_{x}^{2}-\left\{R _ { 1 } \left(\sigma R_{1}+\right.\right.\right.} \\
& \Omega \mathrm{T} 2 k z 2 Q V 2 k 2 A+2 \Omega z 2+k x 2 \Omega T 2 k 24 R 1 \Omega z 2+d R 1 k 2 V 2 A R 1+ \\
& k z 2 V 2 d A+4 d k 2 V 2 \Omega x 2 A+R 1 k z 4 Q 2 V 4 k 4 A 2+4 k z 2 Q V 2 k 2 \Omega z R 1 A \quad+4 k z 2 \quad k 2 R 1+k 2 V 2 d A \Omega x 2 \\
& \Omega T 2-2 \Omega x k x k 2 \Omega T 22 \Omega z k 2 R 1+k 2 V 2 d A+V 2 k z 2 Q A R 1+k 2 V 2 d A-d k z 2 Q V 4 k 2 A 2-2 \Omega z d V 2 A+V 2 k \\
& z 2 R 1 Q A+2 R 1 \Omega z k 2=0 .
\end{aligned}
$$

The dispersion relation (24) shows a general dispersion relation for wave propagation in an homogeneous self-gravitating partially ionized plasma incorporating the effect of rotation, thermal conductivity, 
radiative heat-loss function, Hall current, electrical resistivity and viscosity of the medium. We find that in this dispersion relation the terms due to the Hall current have entered through the factor $Q$. Now, we will reduce the dispersion relation (24) in two different modes of propagation, parallel and perpendicular to the magnetic field for finding the effect of considered parameter, separately.

The above dispersion relation is the modified form of the dispersion relation obtained by Dangarh et al. [28] due the consideration of Hall current, rotation, viscosity and electrical resistivity, excluding electron inertia. Also ignoring the effect of rotation, Hall current and neutral particles the above dispersion relation reduces to Kaothekar and Chhajlani [29] excluding permeability and FLR correction. Again equation (24) gives the same result as Bora and Talwar [30] by ignoring rotation and viscosity and adding the effect of electron inertia in that case.

\section{Analysis of Dispersion Relation}

Now we will discuss the dispersion relation (24) for the following two modes of propagation, parallel and perpendicular to the magnetic field.

\subsection{Longitudinal Mode of Propagation}

For this case we assume all the perturbations longitudinal to the direction of the magnetic field i.e. $\left(k_{z}=\right.$ $\left.k, k_{x}=0\right)$. This is the dispersion relation reduces in the simple form to give.

$$
\begin{aligned}
& -\left\{\left(R_{1}+\frac{k^{2} V^{2} d}{A}\right)\left(R_{1}+\frac{k^{2} V^{2} d}{A}\right)\left(\sigma R_{1}+\Omega_{\mathrm{T}}^{2}\right) R_{1}\right\}-4\left(R_{1}+\frac{k^{2} V^{2} d}{A}\right)\left(\sigma R_{1}+\Omega_{\mathrm{T}}^{2}\right) \Omega_{x}^{2}-\left\{R _ { 1 } \left(\sigma R_{1}+\right.\right. \\
& \Omega \mathrm{T} 2 Q V 2 k 4 A+2 \Omega z 2+4 R 1+k 2 V 2 d A \Omega \times 2 \Omega T 2=0 .
\end{aligned}
$$

Equation (25) gives the general dispersion relation for an infinite homogeneous, uniformly magnetized self-gravitating viscous partially ionized plasma having Hall current, finite electrical resistivity and thermal conductivity incorporated rotation, radiative heat-loss function, when the disturbances are propagating parallel to the magnetic field. Again for simplicity, the dispersion relation (25) is discussed for axis of rotation is along and perpendicular to the magnetic field separately.

\subsubsection{Axis of rotation along magnetic field}

When the axis of rotation is along the magnetic field, we put $\Omega_{x}=0$ and $\Omega_{z}=\Omega$, the dispersion relation $(25)$ reduces to

$$
-R_{1}\left(\sigma R_{1}+\Omega_{\mathrm{T}}^{2}\right)\left(\left(R_{1}+\frac{k^{2} V^{2} d}{A}\right)\left(R_{1}+\frac{k^{2} V^{2} d}{A}\right)+4 \Omega^{2}+\frac{k^{4} Q^{2} V^{4} k^{4}}{A^{2}}+4 \Omega \frac{Q V^{2} k^{4}}{A}\right)=0 .
$$

This is the reduced dispersion relation for a wave propagating in longitudinal direction when axis of rotation is along the magnetic field. We find that the dispersion relation (26) is modified due to the presence of neutral particles, thermal conductivity, radiative heat-loss functions, Hall current, viscosity of medium and electrical resistivity. This dispersion relation (26) has three independent factors; each represents the modes of propagation incorporating different parameters. The first factor of equation (26) equal to zero and putting the value of $R_{1}$ we get.

$$
\sigma^{2}+\sigma F+\Omega_{\mathrm{v}} v_{c}=0 .
$$

where $F=\left\{v_{c}(1+B)+\Omega_{\mathrm{v}}\right\}$. Equation (27) cannot have a real positive root hence it satisfies the necessary and sufficient condition of stability. Thus equations (27) represents stable damped mode due to viscosity of medium, modified by the effect of collision frequency. This equation is same as Chhajlani and Parihar [31] and Kaothekar and Chhajlani [29]. It is evident from the equation (27) that the condition of stability of the fluid is independent of the magnetic field, electrical resistivity, thermal conductivity, selfgravitation, radiative heat-loss function and Hall current. The second factor of equation (26) on simplifying, by putting the value of $\Omega_{\mathrm{T}}^{2}$, and $R_{1}$ gives.

$$
\sigma^{4}+\sigma^{3}(F+\beta)+\sigma^{2}\left[v_{c} \Omega_{\mathrm{v}}+\Omega_{\mathrm{j}}^{2}+F \beta\right]+\sigma\left[v_{c}\left\{\Omega_{\mathrm{j}}^{2}+\beta \Omega_{\mathrm{v}}\right\}+\Omega_{\mathrm{I}}^{2}\right]+v_{c} \Omega_{\mathrm{I}}^{2}=0 .
$$

This dispersion relation for self-gravitating fluid incorporated effect of neutral particles, viscosity, thermal conductivity, Hall current and radiative heat-loss function. It is evident from equation (28) that the condition of instability is independent of magnetic field, rotation and electrical effects such as Hall current and electrical resistivity. The dispersion relation (28) is a fourth degree equation which may be reduced to particular cases so that the effect of each parameter is analyzed separately. If we neglect the effect of viscosity $v=0$ we get,

$$
\sigma^{4}+\sigma^{3}\left[v_{c}(1+B)+\beta\right]+\sigma^{2}\left[v_{c}(1+\mathrm{B}) \beta+\Omega_{\mathrm{j}}^{2}\right]+\sigma\left[v_{c} \Omega_{\mathrm{j}}^{2}+\Omega_{\mathrm{I}}^{2}\right]+v_{c} \Omega_{\mathrm{I}}^{2}=0 .
$$

Equation (29) admits at least one positive root corresponding to the instability of the system. The constant term of this dispersion relation, thus the conditions of instability for this case is given as

$$
k^{2}(\gamma-1)\left(\mathcal{L}_{T} T-\mathcal{L}_{\rho} \rho+\frac{\lambda k^{2} T}{\rho}\right)-4 \pi G \rho(\gamma-1)\left(\frac{\mathcal{L}_{T} T \rho}{p}+\frac{\lambda k^{2} T}{p}\right)<0 .
$$


The above inequality can be solved to yield the following critical wave number

$$
k_{j 1}=\frac{1}{2}\left[a_{1} \pm\left\{a_{1}^{2}+b_{1}\right\}^{1 / 2}\right] .
$$

where $a_{1}=\left\{\frac{4 \pi G \rho}{C^{\prime 2}}+\frac{\rho^{2} \mathcal{L}_{\rho}}{\lambda T}-\frac{\rho \mathcal{L}_{T}}{\lambda}\right\}, b_{1}=\left\{\frac{16 \pi G \rho^{2} \mathcal{L}_{T}}{\lambda C^{2}}\right\}$. and $C^{\prime}=\sqrt{p / \rho}$ is the isothermal velocity of the sound. It is evident from equation (31) the medium represented by equation (29) is unstable for all wave number $k<k_{j 1}$. It may be noted here that the critical wave number involves derivatives of temperature dependent and density dependent heat-loss function and coefficient of thermal conductivity of thermally conducting medium.

This condition of instability (30) is same as the condition of radiative instability earlier obtained by Bora and Talwar [30] and also Kaothekar and Chhajlani [29]. Again if we reduce equation (29) for fully ionized plasma $\left(v_{c}=0\right)$ as

$$
\sigma^{3}+\sigma^{2} \beta+\sigma \Omega_{\mathrm{j}}^{2}+\Omega_{\mathrm{I}}^{2}=0 .
$$

From equation (32), it is clear that the system will leads to the instability, when

$$
k^{2}(\gamma-1)\left(\mathcal{L}_{T} T-\mathcal{L}_{\rho} \rho+\frac{\lambda k^{2} T}{\rho}\right)-4 \pi G \rho(\gamma-1)\left(\frac{\mathcal{L}_{T} T \rho}{p}+\frac{\lambda k^{2} T}{p}\right)<0 .
$$

This condition of instability (33) is same as the condition of instability (30) for a partially ionized system. Now on comparing equation (30) and (33) we notice that in both cases, for partially ionized plasma and for fully ionized plasma we get the same condition of instability, it means that the condition of radiative instability is independent of effect of collision frequency. Again the comparison of equation (29) and (32) reveals that the constant term of equation (32) is independent of the effect of collision frequency while the effect of collision frequency is multiplied with constant term of equation (29). It means that the presence of neutral particle does not contribute in the condition of instability but modified dispersion relation as well as the growth rate of instability for partially ionized medium.

For non-radiating but thermally conducting, viscous and self-gravitating fluid having neutral particles, the dispersion relation $(28)$ reduces to

$\sigma^{4}+\sigma^{3}\left(F+\frac{\lambda \gamma k^{2}}{\rho c_{p}}\right)+\sigma^{2}\left[\Omega_{\mathrm{v}} v_{c}+F \frac{\lambda \gamma k^{2}}{\rho c_{p}}+\Omega_{j 1}^{2}\right]+\sigma\left[v_{c}\left(\Omega_{\mathrm{j} 1}^{2}+\Omega_{\mathrm{v}} \frac{\lambda \gamma k^{2}}{\rho c_{p}}\right)+\frac{\lambda \gamma k^{2}}{\rho c_{p}} \Omega_{j 1}^{2}\right]+v_{c} \Omega_{j 1}^{2} \frac{\lambda \gamma k^{2}}{\rho c_{p}}=0$.

where $C^{\prime}=\left(\frac{p}{\rho}\right)^{1 / 2}$ is the isothermal velocity of the sound.

$$
\Omega_{j 1}^{2}=C^{2} k^{2}-4 \pi G \rho .
$$

It is clear from the constant term of equation (34) that the system leads to instability if $\Omega_{\mathrm{j} 1}^{2}<0$, which gives

$$
\begin{aligned}
& C^{2} k^{2}-4 \pi G \rho<0 . \quad \text { or } \\
& k_{j 2}=\left(\frac{4 \pi G \rho}{C^{2}}\right)^{1 / 2} .
\end{aligned}
$$

where $k_{j 2}$ is the modified Jeans wave number for thermally conducting system. Again if the system is thermally non-conducting, non-radiating but self gravitating i.e. $\alpha=\beta=v_{c}=0$ then the dispersion relation (28) reduces to

$$
\sigma^{2}+\sigma \Omega_{\mathrm{v}}+\Omega_{\mathrm{j}}^{2}=0 \text {. }
$$

It is clear from equation (37) that when $\Omega_{j}^{2}<0$, the product of the roots of equation (37) must, therefore, be negative. This implies that at least one root of $\sigma$ is positive. Hence, the system is unstable. Thus, for the cases of equation (37) the condition of instability is

$$
\begin{aligned}
& \Omega_{j}^{2}=\left(C^{2} k^{2}-4 \pi G \rho\right)<0 . \\
& k<k_{j}=\left(\frac{4 \pi G \rho}{C^{2}}\right)^{1 / 2} .
\end{aligned}
$$

where $k_{j}$ is the Jeans wave number. Equation (38) is original Jeans expression for instability. Now if $\Omega_{\mathrm{I}}^{2}>0$ and $\Omega_{\mathrm{j}}^{2}>0$, then all the coefficient of the equation (28) are positive, which is a necessary condition for the stability of the system. To obtain the sufficient condition, the principal diagonal minors of the Hurwitz must be positive. We calculate the minors and get

$$
\begin{aligned}
& \Delta_{1}=(F+\beta)>0 . \text { as } \gamma>1 \\
& \Delta_{2}=\left[F \Omega_{\mathrm{v}} v_{c}+\beta v_{c} \Omega_{\mathrm{j}}^{2}+\Omega_{\mathrm{v}} \Omega_{\mathrm{j}}^{2}+\beta \Omega_{\mathrm{j}}^{2}+F \beta^{2}+F^{2} \beta-\Omega_{\mathrm{I}}^{2}\right]>0 . \\
& \Delta_{3}=\left[F \beta \Omega_{\mathrm{v}}^{2} v_{c}^{2}+v_{c}^{2} \Omega_{\mathrm{v}} F \Omega_{\mathrm{j}}^{2}+F \Omega_{\mathrm{v}} v_{c} \Omega_{\mathrm{I}}^{2}+F^{2} v_{c} \Omega_{\mathrm{v}} \beta^{2}+F^{2} v_{c} \beta \Omega_{\mathrm{j}}^{2}+F^{2} \Omega_{\mathrm{I}}^{2} \beta+F v_{c} \Omega_{\mathrm{v}} \beta^{3}+\right. \\
& \nu c \beta 2 \Omega \mathrm{j} 2 F+v c \Omega \mathrm{v} \beta 2 \Omega \mathrm{j} 2+\nu c \beta \Omega \mathrm{j} 4+\Omega \mathrm{j} 2 \Omega \mathrm{I} 2 \beta+B \beta \nu c 2 \Omega \mathrm{v} \Omega \mathrm{j} 2+\beta \Omega \mathrm{j} 2 \nu c \Omega \mathrm{v} 2+\Omega \mathrm{j} 2 \Omega \mathrm{I} 2 B \nu c+\Omega \mathrm{j} 2 \Omega \mathrm{I} 2 \Omega \mathrm{v} \\
& +\nu c B \Omega \mathrm{j} 4+\nu c \Omega \mathrm{v} \Omega \mathrm{j} 4+\nu c B \beta 2 \Omega \mathrm{I} 2+\Omega \mathrm{v} \beta 2 \Omega \mathrm{I} 2-\Omega \mathrm{I} 2 \nu c \Omega \mathrm{j} 2+\Omega \mathrm{I} 2+\Omega \mathrm{v} \nu c \beta+r 12 v c+2 r 1 \nu c \beta>0 . \\
& \Delta_{4}=v_{c} \Omega_{\mathrm{I}}^{2} \Delta_{3}>0 .
\end{aligned}
$$

These all $\Delta$ 's are positive, thereby, satisfying the Routh-Hurwitz criterion, Hence, the system expressed by equation (28) is stable if $\Omega_{j}^{2}>0$ and $\Omega_{I}^{2}>0$. Now equating zero the third factor of equation (26) and after solving we obtain dispersion relation as. 
$E_{1}=\left(4 \Omega_{m}+2 F\right)$.

$E_{2}=\left\{\Omega_{m}\left(6 \Omega_{m}+8 F\right)+2 Q^{2} k^{4}+2 \Omega_{\mathrm{v}} v_{c}+F^{2}+2 V^{2} k^{2}+4 \Omega^{2}\right\}$.

$E_{3}=\left\{\Omega_{m}\left(12 F \Omega_{m}+4 \Omega_{m}^{2}+4 Q^{2} k^{4}+4 F^{2}+6 V^{2} k^{2}+16 \Omega^{2}+8 \Omega_{\mathrm{v}} v_{c}\right)+v_{c}\left(2 \Omega_{\mathrm{v}} F+8 \Omega^{2}+2 V^{2} k^{2}\right)+\right.$ $4 F Q 2 k 4+2 F V 2 k 2$.

$E_{4}=\left\{\Omega_{m}\left(8 F \Omega_{m}^{2}+\Omega_{m}^{3}+2 Q^{2} k^{4} \Omega_{m}+8 F Q^{2} k^{4}+6 \Omega_{m} F^{2}+6 \Omega_{m} V^{2} k^{2}+6 F V^{2} k^{2}+24 \Omega_{m} \Omega^{2}\right)+\right.$ $\Omega m v c 12 \Omega m \Omega v+8 \Omega v F+32 \Omega 2+6 V 2 k 2+v c 2 F V 2 k 2+4 Q 2 k 4 \Omega v+\Omega v 2 v c+2 \Omega v V 2 k 2+4 v c \Omega 2+Q 2 k 4 Q 2 k 4+2$ $F 2+2 V 2 k 2+8 \Omega 2+V 4 k 4+4 \Omega V 2 k 4 Q$.

$E_{5}=\left\{\Omega_{m}\left(2 F \Omega_{m}^{3}+4 F Q^{2} k^{4} \Omega_{m}+4 F^{2} Q^{2} k^{4}+4 \Omega_{m}^{2} F^{2}+7 F \Omega_{m} V^{2} k^{2}+2 \Omega_{m}^{2} V^{2} k^{2}+2 Q^{2} k^{4} V^{2} k^{2}+2 V^{4} k^{4}+\right.\right.$ $16 \Omega m 2 \Omega 2+16 \Omega 2 Q 2 k 4+8 \Omega Q V 2 k 4+\Omega m v c 8 \Omega v \Omega m 2+6 \Omega m V 2 k 2+8 \Omega v Q 2 k 4+12 F \Omega m \Omega v+4 \Omega v 2 v c+6 F V 2$ $k 2+6 \Omega v V 2 k 2+16 v c \Omega 2+48 \Omega m \Omega 2+v c 4 F Q 2 k 4 \Omega v+2 V 2 k 2 \Omega v v c+2 Q 2 k 4 V 2 k 2+2 V 4 k 4+16 Q 2 k 4 \Omega 2+8 \Omega Q$ $V 2 k 4+Q 2 k 42 F Q 2 k 4+2 F V 2 k 2$.

$E_{6}=\left\{\Omega_{m} v_{c}\left(2 \Omega_{\mathrm{v}} \Omega_{m}^{3}+4 \Omega_{\mathrm{v}} Q^{2} k^{4} \Omega_{m}+Q^{2} k^{4} V^{2} k^{2}+8 F \Omega_{\mathrm{v}} v_{c} \Omega_{m}+6 F \Omega_{m} V^{2} k^{2}+8 F Q^{2} k^{4} \Omega_{\mathrm{v}}+6 \Omega_{\mathrm{v}}^{2} v_{c} \Omega_{m}+\right.\right.$ $6 V 2 k 2 v c \Omega v+2 \Omega m 2 V 2 k 2+5 \Omega \mathrm{v} \Omega m V 2 k 2+4 V 4 k 4+V 2 k 2 Q 2 k 4+32 \Omega m 2 \Omega 2+24 \Omega m v c \Omega 2+32 Q 2 k 4 \Omega 2+16$ $\Omega Q V 2 k 4+\Omega m F 2 \Omega m 3+2 F 2 Q 2 k 4 \Omega m+2 F \Omega m 2 V 2 k 2+2 F Q 2 k 4 V 2 k 2+2 \Omega m V 4 k 4+4 \Omega m 2 \Omega 2+8 \Omega 2 Q 2 k 4 \Omega m$ $+4 Q V 2 k 4 \Omega m \Omega+\nu c 2 Q 2 k 4 \Omega v 2 v c+2 \Omega v Q 2 k 4 V 2 k 2+2 F Q 2 k 4 V 2 k 2+V 4 k 4 v c+2 \Omega v Q 4 k 8+8 \Omega 2 Q 2 k 4 v c+4 \Omega$ $Q V 2 k 4 v c+Q 2 k 4 F 2 Q 2 k 4+V 4 k 4+4 \Omega Q V 2 k 4+4 \Omega 2 Q 2 k 4$.

$E_{7}=\left\{\Omega_{m} v_{c}\left(2 F \Omega_{\mathrm{v}} \Omega_{m}^{3}+4 F \Omega_{\mathrm{v}} Q^{2} k^{4} \Omega_{m}+2 F \Omega_{m}^{2} V^{2} k^{2}+2 F Q^{2} k^{4} V^{2} k^{2}+4 \Omega_{m}^{2} \Omega_{\mathrm{v}}^{2} v_{c}+6 V^{2} k^{2} \Omega_{m} v_{c} \Omega_{\mathrm{v}}+\right.\right.$ $4 Q 2 k 4 \Omega v 2 v c+2 \Omega m 2 \Omega v V 2 k 2+2 V 2 k 2 Q 2 k 4 \Omega v+3 \Omega m V 4 k 4+2 V 4 k 4 v c+16 \Omega m 2 \Omega 2 v c+16 Q 2 k 4 \Omega 2 v c+8 \Omega m$ $3 \Omega 2+16 Q 2 k 4 \Omega 2 \Omega m+8 v c \Omega v 2 Q V 2 k 4+8 \Omega \Omega m Q V 2 k 4+v c 2 F Q 4 k 8 \Omega v+2 \Omega v v c Q 2 k 4 V 2 k 2+8 \Omega 2 Q 4 k 8+2 Q 2$ $k 8 V 4+8 \Omega Q V 2 k 4 Q 2 k 4$.

$E_{8}=\left\{\Omega_{m} v_{c}\left(v_{c} \Omega_{\mathrm{v}}^{2} \Omega_{m}^{3}+2 Q^{2} k^{4} \Omega_{m} \Omega_{\mathrm{v}}^{2} v_{c}+\Omega_{m}^{2} V^{2} k^{2} v_{c} \Omega_{\mathrm{v}}+2 v_{c} \Omega_{\mathrm{v}} Q^{2} k^{4} V^{2} k^{2}+\Omega_{m}^{2} v_{c} \Omega_{\mathrm{v}} V^{2} k^{2}+V^{4} k^{4} \Omega_{m} v_{c}+\right.\right.$ $4 \Omega m 3 \Omega 2 v c+4 \Omega Q k 4 V 2 \Omega m v c+8 \Omega m Q 2 k 4 \Omega 2 v c+v c v c \Omega v 2 Q 4 k 8+4 \Omega 2 Q 4 k 8 v c+4 \Omega Q v 2 k 4 Q 2 k 4 v c+Q 2 k 8 V$ $4 \nu c$.

The dispersion relation (39) consisted with the terms showing the effect of magnetic field, viscosity, finite electrical resistivity, rotation, Hall current and the effect of the neutral particles, but it does not involve terms of thermal conductivity, radiative heat-loss function and self-gravitation. Since the coefficients of the equation (39) are all positive including the constant term, therefore, this equation cannot have a positive root, which means that the system represented by equation (39) is stable. Thus, this equation gives Alfven modes modified by the dissipative effects of viscosity, finite electrical resistivity, Hall current and neutral particles and rotation.

In the absence of neutral particle, Hall current, finite electrical resistivity i.e. [ $\left.v_{c}=Q=\Omega_{\mathrm{m}}=0\right]$, thus, the dispersion relation reduces to

$$
\sigma^{4}+\sigma^{3} 2 \Omega_{\mathrm{v}}+\sigma^{2}\left[\Omega_{\mathrm{v}}^{2}+2 V^{2} k^{2}+4 \Omega^{2}\right]+\sigma\left[2 \Omega_{\mathrm{v}} V^{2} k^{2}\right]+V^{4} k^{4}=0 .
$$

The necessary condition for stability of the system is that the equation (40) should have all coefficients positive, which is satisfied. The sufficient condition is that the Routh-Hurwitz criterion must be satisfied, according to which all the principal diagonal minors of the Hurwitz matrix must be positive for a stable system. For the fourth-degree equation (40) Hurwitz matrixes are as shown below.

$$
\begin{aligned}
& \Delta_{1}=2 \Omega_{\mathrm{v}}>0 . \\
& \Delta_{2}=2 \Omega_{\mathrm{v}}\left[\Omega_{\mathrm{v}}^{2}+2 V^{2} k^{2}+4 \Omega^{2}\right]>0 . \\
& \Delta_{3}=4 \Omega_{\mathrm{v}}^{2} V^{2} k^{2}\left[\Omega_{\mathrm{v}}^{2}+4 \Omega^{2}\right]>0 . \\
& \Delta_{4}=V^{4} k^{4} \Delta_{3}>0 .
\end{aligned}
$$

We find that all $\Delta$ 's are positive, which shows that a magnetized viscous plasma with finite electrical conductivity is stable. Thus, the equation (40) represents a stable Alfven mode modified by the dissipative effect of viscosity, and finite electrical resistivity. It may be remarked that for non-rotating, the Hurwitz's criterion is also satisfied which means that non-rotating is also stable system.

\subsubsection{Axis of rotation perpendicular to the magnetic field}

In the case of a rotation axis perpendicular to the magnetic field we put $\Omega_{\mathrm{x}}=\Omega$, and $\Omega_{\mathrm{z}}=0$ in the dispersion relation $(25)$ and this gives.

$$
-R_{1}\left[\left(R_{1}+\frac{k^{2} V^{2} d}{A}\right)\left(R_{1}+\frac{k^{2} V^{2} d}{A}\right)\left(\sigma R_{1}+\Omega_{\mathrm{T}}^{2}\right)+\left(\sigma R_{1}+\Omega_{\mathrm{T}}^{2}\right)\left(\frac{V^{4} k^{4} k^{4} Q^{2}}{\mathrm{~A}^{2}}\right)+4 \sigma \Omega^{2}\left(R_{1}+\frac{k^{2} V^{2} d}{A}\right)\right]=0 .
$$

The above equation represents the longitudinal wave propagation through viscous, magnetized partially ionized Hall Plasma having the effect of rotation, electrical resistivity, radiation with self-gravitation, when the 
axis of rotation is perpendicular to the magnetic field. This dispersion relation is the product of two independent factors. These factors show the mode of propagations incorporating different parameters as discussed below. The first factors of equation (41) is obtained

$$
\sigma^{2}+\sigma F+\Omega_{\mathrm{v}} v_{c}=0
$$

We have been already discussed in equation (27). The second factor of (41) is equal to zero,

$$
\left[\left(R_{1}+\frac{k^{2} V^{2} d}{A}\right)\left(R_{1}+\frac{k^{2} V^{2} d}{A}\right)\left(\sigma R_{1}+\Omega_{\mathrm{T}}^{2}\right)+\left(\sigma R_{1}+\Omega_{\mathrm{T}}^{2}\right)\left(\frac{V^{4} k^{4} k^{4} \mathrm{Q}^{2}}{\mathrm{~A}^{2}}\right)+4 \sigma \Omega^{2}\left(R_{1}+\frac{k^{2} V^{2} d}{A}\right)\right]=0 .
$$

On substituting the values of $\mathrm{A}, \mathrm{d}, \Omega_{\mathrm{T}}^{2}$ and $R_{1}$ gives, the following twelfth order polynomial equation $\sigma^{12}+\sigma^{11} C_{1}+\sigma^{10} C_{2}+\sigma^{9} C_{3}+\sigma^{8} C_{4}+\sigma^{7} C_{5}+\sigma^{6} C_{6}+\sigma^{5} C_{7}+\sigma^{4} C_{8}+\sigma^{3} C_{9}+\sigma^{2} C_{10}+\sigma C_{11}+C_{12}=0$. (44)

The coefficient of dispersion relation is very lengthy its constant term of the last coefficients gives the condition of instability.

$$
\begin{aligned}
& \Omega_{I}^{2}\left[v_{c}^{3} \Omega_{\mathrm{v}} \Omega_{m}\left(\Omega_{\mathrm{v}} \Omega_{m}^{3}+2 \Omega_{\mathrm{v}} \Omega_{m} Q^{2} k^{4}+2 V^{2} k^{2} \Omega_{m}^{2}+2 V^{2} k^{2} Q^{2} k^{4}\right)+v_{c}^{3}\left(Q^{4} k^{8} \Omega_{\mathrm{v}}^{2}+\right.\right. \\
& V 4 k 4 \Omega m 2+V 4 k 8 Q 2=0 .
\end{aligned}
$$

Equation (44) represents the general dispersion relation for an infinite homogeneous, thermally conducting, self-gravitating, viscous uniformly magnetized partially ionized plasma, incorporating Hall current, and radiative heat-loss function, when the disturbances are propagating along the direction of magnetic field and the axis of rotation is perpendicular to the direction of magnetic field. The condition of instability is obtained from constant term of equation (44) and gives as

$$
k^{2}(\gamma-1)\left(\mathcal{L}_{T} T-\mathcal{L}_{\rho} \rho+\frac{\lambda k^{2} T}{\rho}\right)-4 \pi G \rho(\gamma-1)\left(\frac{\mathcal{L}_{T} T \rho}{p}+\frac{\lambda k^{2} T}{p}\right)<0 .
$$

The above condition of instability is identical to the condition (30) we find that the condition of instability for this mode of propagations in both the cases of rotation is the same and there is no effect of the rotation on this condition of instability. Also we notice that the condition of instability for longitudinal mode of propagation is same and independent of Hall current, electrical resistivity, magnetic field and the effect of neutral-ion collision. Hence we conclude that for longitudinal direction of propagation the radiative instability criterion does not depend on Hall current, electrical resistivity and magnetic field, either axis of rotation taking along the magnetic field or perpendicular to magnetic field.

\subsection{Transverse Mode of Propagation}

For this case we assume all the perturbations are propagating perpendicular to the direction of the magnetic field, for, our convenience, we take $\boldsymbol{k}_{x}=\boldsymbol{k}$, and $\boldsymbol{k}_{z}=\mathbf{0}$, the general dispersion relation (24) reduces to

$$
-\left\{\sigma R_{1}^{4}+\sigma R_{1}^{3} \frac{V^{2} k^{2}}{d}+R_{1}^{3} \Omega_{T}^{2}+\sigma 4 R_{1}^{2} \Omega_{x}^{2}+4 \Omega_{x}^{2} R_{1} \Omega_{T}^{2}+\frac{V^{2} k^{2}}{d} 4 \sigma R_{1} \Omega_{x}^{2}+4 R_{1}^{2} \Omega_{z}^{2} \sigma\right\}=0 .
$$

The dispersion relation (46) shows the influence of viscosity, rotation, on self-gravitating partiallyionized plasmas with radiative heat-loss function, electrical resistivity and thermal conductivity. In the absence of viscosity, rotation, partially-ionized plasmas, (46) reduces to that of Bora and Talwar [30] in dimensional form. Now we discuss this dispersion relation (46) in the case of rotation axes parallel and perpendicular to the magnetic field.

\subsubsection{Axis of rotation along magnetic field}

When the axis of rotation is along the magnetic field, we put $\Omega_{\mathrm{x}}=0$, and $\Omega_{\mathrm{z}}=\Omega$ in the dispersion relation (46) and this gives.

$$
R_{1}^{2}\left(\sigma R_{1}^{2}+\sigma R_{1} \frac{V^{2} k^{2}}{d}+R_{1} \Omega_{T}^{2}+4 \sigma \Omega^{2}\right)=0 .
$$

This equation represents the effect of thermal conductivity, magnetic field, radiative heat-loss function, rotation, gravitational attraction and finite electrical resistivity on the presence of neutral particles. Equation (47) has two independent factors, each representing a different mode of propagation. The first factor of equation (47) gives, on substituting the values of $\mathrm{R}_{1}$ gives,

$$
\sigma^{2}+\sigma F+\Omega_{\mathrm{v}} v_{c}=0
$$

Equation (48) is identical with equation (27) and represents a viscous type of damped stable mode modified by the effects of viscosity and collision frequency. The second factor of equation of (47) equal to zero gives

where

$$
\sigma^{7}+A_{1} \sigma^{6}+\sigma^{5} A_{2}+\sigma^{4} A_{3}+\sigma^{3} A_{4}+\sigma^{2} A_{5}+\sigma A_{6}+A_{7}=0 .
$$

$$
\begin{aligned}
A_{1}= & \left(\Omega_{m}+2 F+\beta\right) . \\
A_{2}= & {\left[\Omega_{m}(2 F+\beta)+2 \Omega_{\mathrm{v}} v_{c}+F(F+2 \beta)+V^{2} k^{2}+\Omega_{J}^{2}+4 \Omega^{2}\right] . } \\
A_{3}= & {\left[\Omega_{m}\left(\Omega_{J}^{2}+F^{2}+2 F \beta+4 \Omega^{2}\right)+2 \Omega_{\mathrm{v}} v_{\mathrm{c}}\left(F+\beta+\Omega_{m}\right)+F\left(\Omega_{J}^{2}+F \beta\right)+k^{2} \mathrm{~V}^{2}(F+\beta+\right.} \\
& \mathcal{\imath}+\Omega / 2+\nu \mathrm{c} \Omega / 2+4 \Omega 2 \beta+8 \Omega 2 \imath \mathrm{c} .
\end{aligned}
$$




$$
\begin{aligned}
A_{4}= & {\left[\Omega_{m}\left(\Omega_{I}^{2}+F^{2} \beta+F \Omega_{J}^{2}+v_{\mathrm{c}} \Omega_{J}^{2}+4 \Omega^{2} \beta\right)+\mathrm{V}^{2} k^{2}\left(F \beta+\beta v_{\mathrm{c}}+F v_{\mathrm{c}}\right)+v_{\mathrm{c}} \Omega_{\mathrm{v}}\left(\Omega_{\mathrm{v}} v_{c}+\right.\right.} \\
& 2 F \beta+2 F \Omega m+2 \beta \Omega m+k 2 \mathrm{v} 2+\Omega J 2+F \Omega / 2+\nu \mathrm{c} F \Omega J 2+\Omega / 2+4 \Omega 2 v c+8 \Omega 2 \beta+ \\
& 8 \Omega 2 \Omega m . \\
A_{5}= & {\left[\Omega_{m}\left(v_{c} \Omega_{I}^{2}+F v_{c} \Omega_{J}^{2}+F \Omega_{I}^{2}\right)+\Omega_{\mathrm{v}} v_{c}\left(\Omega_{\mathrm{v}} v_{c} \Omega_{m}+\Omega_{\mathrm{v}} v_{c} \beta+2 F \beta \Omega_{m}+\beta V^{2} k^{2}+v_{c} V^{2} k^{2}+\right.\right.} \\
& \left.\left.\Omega_{I}^{2}+\Omega_{m} \Omega_{J}^{2}\right)+v_{c}\left(\Omega_{\mathrm{v}} v_{c} \Omega_{J}^{2}+F \beta V^{2} k^{2}+F \Omega_{I}^{2}+4 \Omega^{2} v_{c} \Omega_{m}+4 \Omega^{2} \beta v_{c}+8 \Omega^{2} \beta \Omega_{m}\right)\right] . \\
A_{6}= & {\left[\Omega_{\mathrm{v}} v_{c}\left(\Omega_{m} \beta \Omega_{\mathrm{v}} v_{c}+v_{c} V^{2} k^{2} \beta+\Omega_{m} \Omega_{I}^{2}+v_{c} \Omega_{I}^{2}+\Omega_{m} v_{c} \Omega_{J}^{2}+4 \Omega_{m} \Omega^{2} \beta v_{c}\right)+F v_{c} \Omega_{m} \Omega_{I}^{2}\right] . } \\
A_{7}= & \left(v_{c}^{2} \Omega_{\mathrm{v}} \Omega_{m} \Omega_{I}^{2}\right) .
\end{aligned}
$$

Equation (49) seventh degree equation, where the constant term $\mathrm{A}_{7}$ is given as

$$
A_{7}=\Omega_{I}^{2}\left(v_{c}^{2} \Omega_{\mathrm{v}} \Omega_{m}\right) \text {. }
$$

Equation (49) represents the dispersion relation for transverse waves propagating through infinite homogeneous, self-gravitating, viscous and magnetized plasma having finite electrical resistivity, thermal conductivity, rotation, and radiative effects with neutral particles. The above equation (49) admits at least one real positive root which leads to the instability of the system as

$$
k^{2}(\gamma-1)\left(\mathcal{L}_{T} T-\mathcal{L}_{\rho} \rho+\frac{\lambda k^{2} T}{\rho}\right)-4 \pi G \rho(\gamma-1)\left(\frac{\mathcal{L}_{T} T \rho}{p}+\frac{\lambda k^{2} T}{p}\right)<0 .
$$

The above condition of instability is same as Bora and Talwar [30] and Kaothekar and Chhajlani [29] for electrical conducting partially ionized plasma in transverse direction of propagation and also same as condition of instability (30). In the absence of viscosity and collision frequency the above dispersion relation reduces in the form

$$
\begin{aligned}
& \sigma^{4}+\sigma^{3}\left(\Omega_{m}+\beta\right)+\sigma^{2}\left[\Omega_{m} \beta+V^{2} k^{2}+\Omega_{J}^{2}+4 \Omega^{2}\right]+\sigma\left[\Omega_{m}\left(\Omega_{J}^{2}+4 \Omega^{2}\right)+k^{2} \mathrm{~V}^{2} \beta+\Omega_{I}^{2}+\right. \\
& 4 \Omega 2 \beta+\Omega m \Omega / 2+4 \Omega 2 \beta=0 .
\end{aligned}
$$

The condition of instability for such case is obtained from the constant term of equation (51), is given as

$$
k^{2}\left(\mathcal{L}_{T} T-\mathcal{L}_{\rho} \rho+\frac{\lambda k^{2} T}{\rho}\right)+\left(4 \Omega^{2}-4 \pi G \rho\right)\left(\frac{\mathcal{L}_{T} T \rho}{p}+\frac{\lambda k^{2} T}{p}\right)<0 .
$$

On comparing equation (50) and (52) we note that there is an extra term added in (52), represents the effect of rotation for non-viscous fluid. It means that the presence of viscosity parameter removes the effect of rotation from the condition of instability. Thus we conclude that the contribution of rotation in the condition of radiative instability is only observed when the fluid is in-viscid. Also we notated that effect of collision frequency does not affect the condition of instability but modifies the growth rate of the present system.

Assuming $\left(\rho \gg \rho_{d}\right)$ so that $\mathrm{B}<<1$ and dividing the dispersion relation (49) by $(4 \pi G \rho)^{1 / 2}$, then the dispersion relation can be written in non-dimensional form as

$\sigma^{* 7}+\sigma^{* 6}\left(\Omega_{m}^{*}+2 \Omega_{\mathrm{v}}^{*}+4 v_{c}^{*}+\beta^{*}\right)$

$+\sigma^{* 5}\left[\Omega_{m}^{*}\left(2 \Omega_{\mathrm{v}}^{*}+4 v_{c}^{*}+\beta^{*}\right)+2 v_{c}^{*}\left(3 \Omega_{\mathrm{v}}^{*}+2 v_{c}^{*}+2 \beta^{*}\right)+V^{* 2} k^{* 2}+\Omega_{J}^{* 2}+4 \Omega^{* 2}+2 \beta^{*} \Omega_{\mathrm{v}}^{*}+\Omega_{\mathrm{v}}^{* 2}\right]$

$+\sigma^{* 4}\left[\Omega_{m}^{*}\left(\Omega_{\mathrm{j}}^{* 2}+2 \beta^{*} \Omega_{\mathrm{v}}^{*}+4 \beta^{*} v_{c}^{*}+4 \Omega^{* 2}+4 v_{c}^{* 2}+\Omega_{\mathrm{v}}^{* 2}+4 \Omega_{\mathrm{v}}^{*} v_{c}^{*}\right)+2 \Omega_{\mathrm{v}}^{*} v_{c}^{*}\left(\Omega_{m}^{*}+\Omega_{\mathrm{v}}^{*}+2 v_{c}^{*}+\beta^{*}\right)+\right.$

$V * 2 k * 2 \Omega \mathrm{v} *+3 \nu c *+\beta *+\nu c * 3 \Omega / * 2+4 \beta * \Omega \mathrm{V} *+4 \beta * \nu c *+8 \Omega * 2+\Omega / * 2+4 \Omega * 2+\Omega \mathrm{V} * \Omega / * 2+\Omega \mathrm{V} * \beta *$

$+\sigma^{* 3}\left[\Omega_{m}^{*}\left(\Omega_{I}^{* 2}+4 v_{c}^{* 2} \beta^{*}+\Omega_{\mathrm{v}}^{*} \beta^{*}+4 \beta^{*} v_{c}^{*} \Omega_{\mathrm{v}}^{*}+2 v_{c}^{*} \Omega_{J}^{* 2}+\Omega_{\mathrm{v}}^{*} \Omega_{J}^{* 2}+v_{c}^{*} \Omega_{J}^{* 2}+4 \Omega^{* 2} \beta^{*}\right)+\mathrm{V}^{* 2} k^{* 2}\left(\beta^{*} \Omega_{\mathrm{v}}^{*}+\right.\right.$

$3 \beta * v c *+2 v c * 2+\Omega v * v c *+\Omega v * v c * v c * \Omega v *+2 \beta * \Omega v *+4 v c * \Omega m *+4 \beta * v c *+2 \Omega m * \Omega v *+2 \beta * \Omega m *+2 \beta * \Omega m *+$ $k * 2 \mathrm{~V} * 2+\Omega / * 2+\nu c * 2 v c * \Omega / * 2+\Omega \mathrm{v} * \Omega / * 2+\Omega / * 2+4 \Omega * 2 v c *+8 \Omega * 2 \beta *+8 \Omega * 2 \Omega m *+2 v c * \Omega / * 2+\Omega \mathrm{v} * \Omega / * 2$. $+\sigma^{* 2}\left[\Omega_{m}^{*}\left(2 v_{c}^{* 2} \Omega_{J}^{* 2}+\Omega_{\mathrm{v}}^{*} v_{c}^{*} \Omega_{J}^{* 2}+3 v_{c}^{*} \Omega_{I}^{* 2}+\Omega_{\mathrm{v}}^{*} \Omega_{I}^{* 2}\right)+\Omega_{\mathrm{v}}^{*} v_{c}^{*}\left(v_{c}^{*} \Omega_{m}^{*} \Omega_{\mathrm{v}}^{*}+\Omega_{\mathrm{v}}^{*} v_{c}^{*} \beta^{*}+4 v_{c}^{*} \beta^{*} \Omega_{m}^{*}+2 \Omega_{\mathrm{v}}^{*} \beta^{*} \Omega_{m}^{*}+\right.\right.$ $\beta * V * 2 k * 2+\nu c * V * 2 k * 2+\Omega / * 2+\Omega m * \Omega / * 2+\nu c * \Omega v * v c * \Omega / * 2+2 \nu c * \beta * V * 2 k * 2+\Omega v * \beta * V * 2 k * 2+2 \nu c * \Omega / * 2+$ $\Omega v * \Omega / * 2+4 \Omega * 2 v c * \Omega m *+4 \Omega * 2 \beta * v c *+8 \Omega * 2 \beta * \Omega m *$

$+\sigma^{*}\left[\Omega_{\mathrm{v}}^{*} v_{c}^{*} \Omega_{m}^{*}\left(\Omega_{m}^{*} \beta^{*} \Omega_{\mathrm{v}}^{*} v_{c}^{*}+v_{c}^{*} V^{* 2} k^{* 2} \beta^{*}+\Omega_{m}^{*} \Omega_{I}^{* 2}+v_{c}^{*} \Omega_{I}^{* 2}+\Omega_{m}^{*} v_{c}^{*} \Omega_{J}^{* 2}+4 \Omega_{m}^{*} \Omega^{* 2} \beta^{*} v_{c}^{*}\right)+2 v_{c}^{* 2} \Omega_{I}^{* 2} \Omega_{m}^{*}+\right.$ $\Omega \mathrm{V} * \nu c * \Omega m * \Omega / * 2$

$$
+\left(v_{c}^{* 2} \Omega_{\mathrm{v}}^{*} \Omega_{m}^{*} \Omega_{I}^{* 2}\right) \text {. }
$$

where the various non dimensional parameters are defined as

$$
\begin{aligned}
& \sigma^{*}=\frac{\sigma}{(4 \pi G \rho)^{1 / 2}}, \quad v_{c}^{*}=\frac{v_{c}}{(4 \pi G \rho)^{1 / 2}}, \quad k^{*}=\frac{k c}{(4 \pi G \rho)^{1 / 2}}, \quad v^{*}=\frac{v(4 \pi G \rho)^{1 / 2}}{C^{2}}, \quad \lambda^{*}=\frac{(\gamma-1) \mathrm{T} \lambda(4 \pi G \rho)^{1 / 2}}{p C^{2}}, \quad \eta^{*}=\frac{\eta(4 \pi G \rho)^{1 / 2}}{C^{2}}, \\
& \Omega^{*}=\frac{\Omega}{(4 \pi G \rho)^{1 / 2}}, \quad \mathrm{~V}^{*}=\frac{\mathrm{v}(4 \pi G \rho)^{1 / 2}}{C}, \quad \mathcal{L}_{\rho}^{*}=\frac{(\gamma-1)_{\rho} \mathcal{L}_{\rho}}{C^{2}(4 \pi G \rho)^{1 / 2}}, \quad \mathcal{L}_{T}^{*}=\frac{(\gamma-1)_{\rho} T \mathcal{L}_{T}}{\rho(4 \pi G \rho)^{1 / 2}}, \quad \Omega_{\mathrm{v}}^{*}=k^{* 2} v^{*}, \quad \Omega_{m}{ }^{*}=\eta^{*} k^{* 2}, \\
& \alpha^{*}=\frac{1}{\gamma}\left(\mathcal{L}_{T}^{*}+\lambda^{*} k^{* 2}\right)-\mathcal{L}_{\rho}^{*}, \quad \beta^{*}=\left(\mathcal{L}_{T}^{*}+\lambda^{*} k^{* 2}\right), \quad \Omega_{I}^{* 2}=\left(k^{* 2} \alpha^{*}-\beta^{*}\right), \quad \Omega_{j}^{* 2}=\left(k^{* 2}-1\right) .
\end{aligned}
$$



The variation of the growth rate $\sigma^{*}$ with wave number $k^{*}$ are shown in fig 1-6

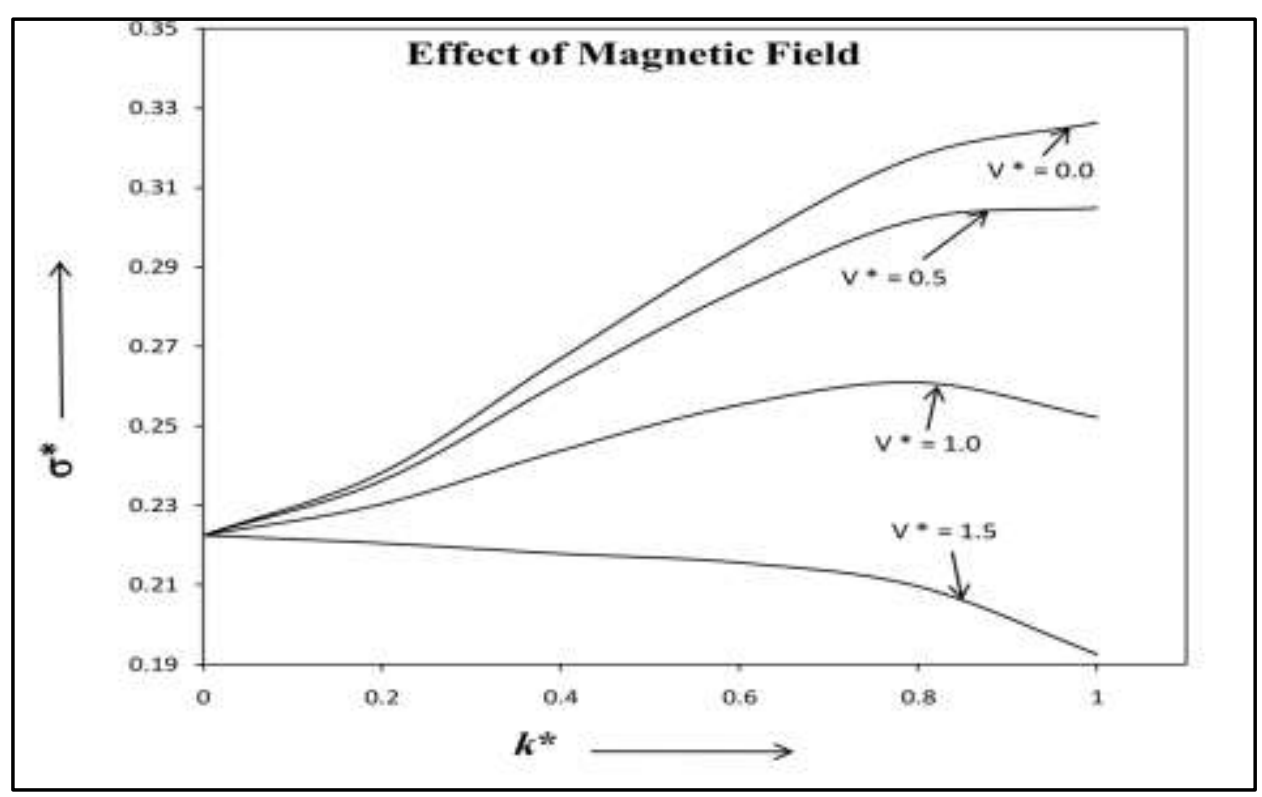

The growth rate is plotted against the non-dimensional wave number $\mathrm{k}^{*}$ with variation in the normalized magnetic effect $V^{*}=0.0,0.5,1.0,1.5$ and the value of other parameter are fixed $\lambda^{*}=v *=v_{c}^{*}=$ $\eta^{*}=\Omega^{*}=1, \mathcal{L}_{T}^{*}=0.0$ and $\mathcal{L}_{\rho}^{*}=0.5$.

From figure (1), we notice that the growth rate of the instability for non-magnetized medium $\left(V^{*}=0\right)$ is higher in comparison with magnetized medium $\left(V^{*}>0\right)$. It is also noted that the value growth rate is decreased with increasing magnetization of medium. Hence, we conclude that the increasing magnetic field tends to stabilize the system.

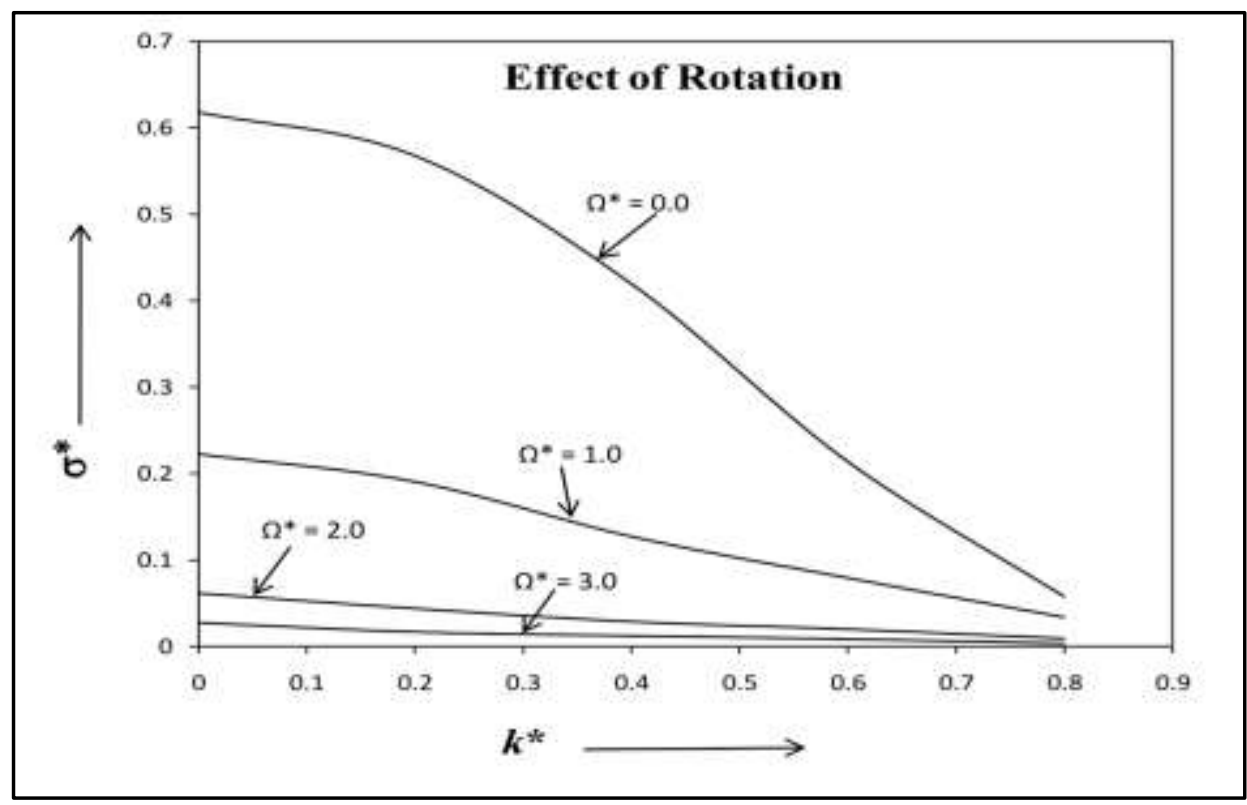

The growth rate is plotted against the non-dimensional wave number $\mathrm{k}^{*}$ with variation in the normalized rotational effect $\Omega^{*}=0.0,1.0,2.0,3.0$ and the value of other parameter are fixed $\lambda^{*}=v^{*}=v_{c}^{*}=\mathrm{V}^{*}$ $=\eta^{*}=1, \mathcal{L}_{T}^{*}=0.0$ and $\mathcal{L}_{\rho}^{*}=-0.5$.

Figure (2), depicts that the growth rate of the instability decreased with increasing values of rotation parameter, for non-rotating medium $\left(\Omega^{*}=0\right)$ the growth rate of the instability is maximum while for higher values of rotation it tends to minimize. Thus, we conclude that rotation parameter reduces the growth rate of the instability and maintain the stability of system. 


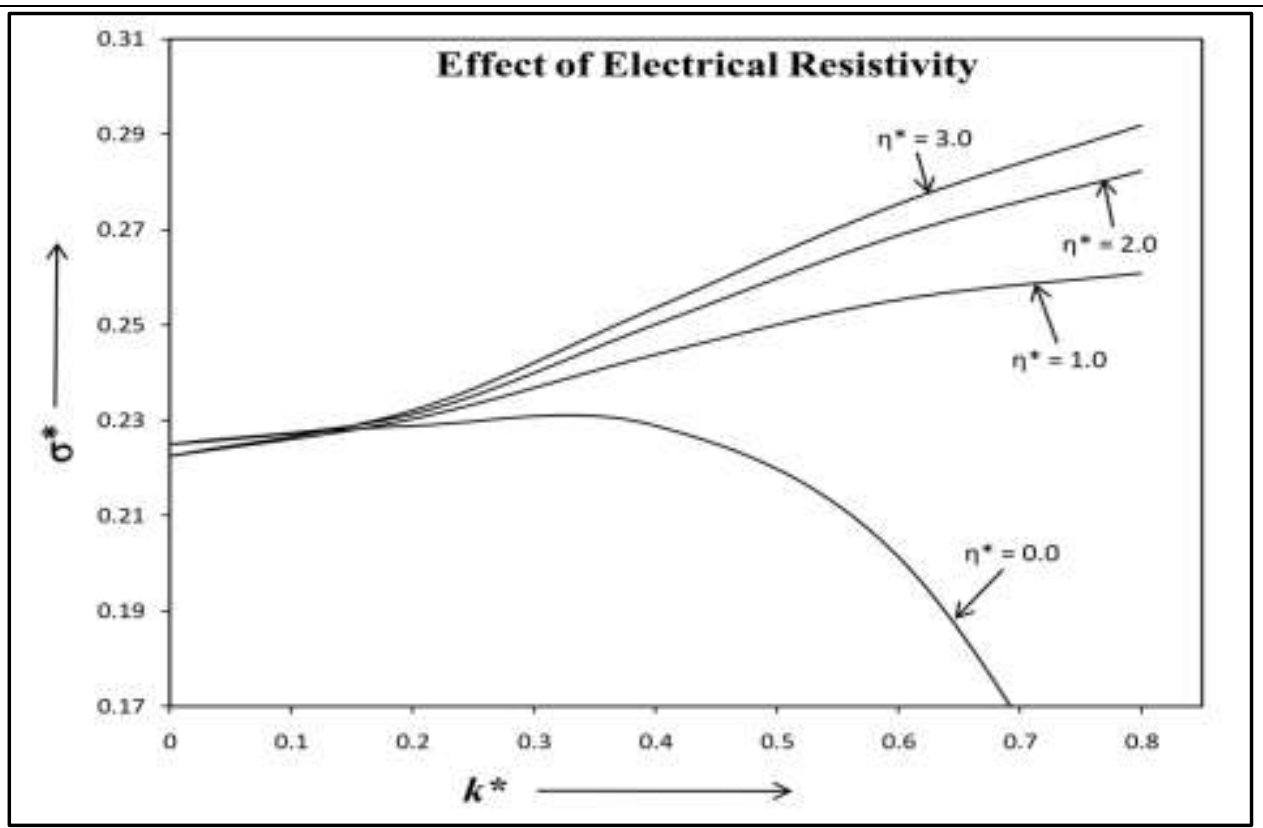

The growth rate is plotted against the non-dimensional wave number $\mathrm{k}^{*}$ with variation in the normalized resistivity effect $\eta^{*}=0.0,1.0,2.0,3.0$ and the value of other parameter are fixed $\lambda^{*}=v^{*}=v_{c}^{*}=\mathrm{V}^{*}$ $=\Omega^{*}=1, \mathcal{L}_{T}^{*}=0.0$ and $\mathcal{L}_{\rho}^{*}=0.5$.

In figure (3) the growth rate of the system is increased with increasing values of electrical resistivity. It means that for the higher values of electrical resistivity, the system tends to get instability. In other words, an infinitely conducting system will be more stable than finitely conducting medium.

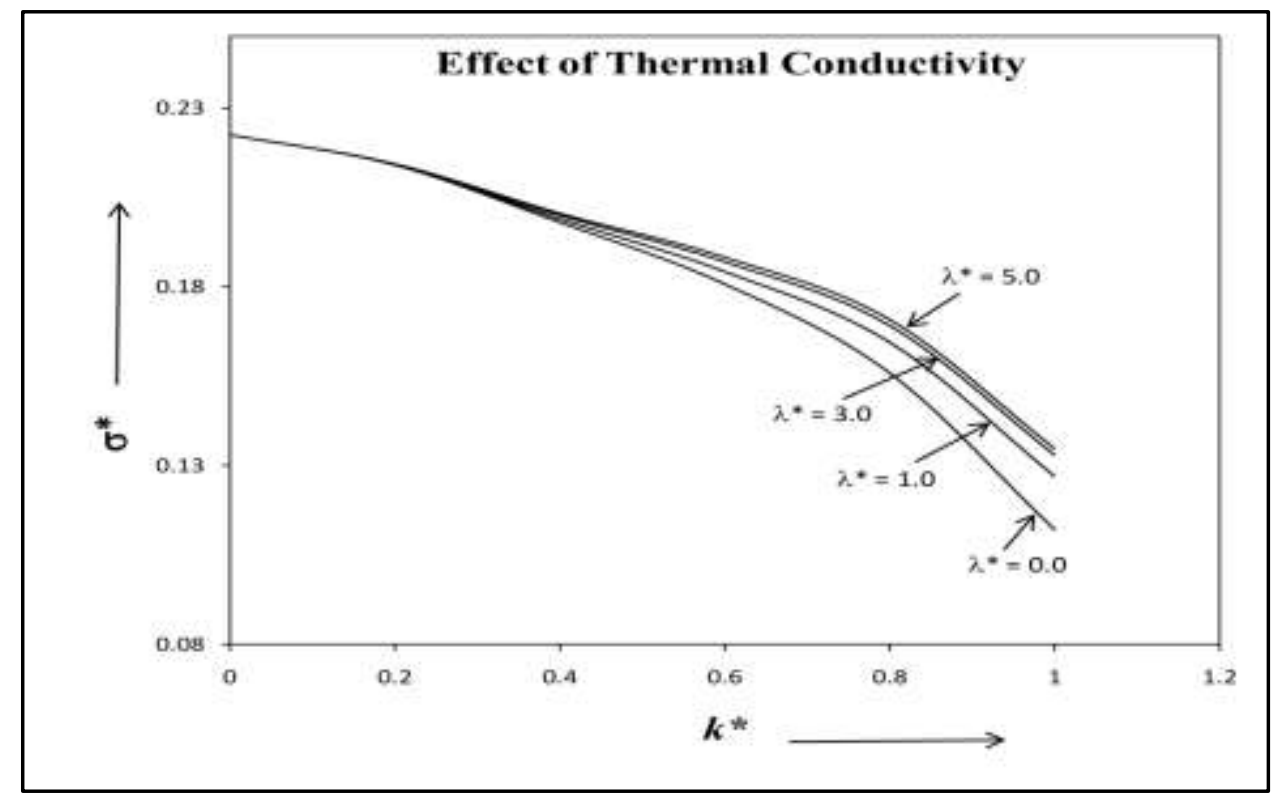

The growth rate is plotted against the non-dimensional wave number $\mathrm{k}^{*}$ with variation in the normalized thermal conductivity effect $\lambda^{*}=0.0,1.0,3.0,5.0$ and the value of other parameter are fixed $\eta^{*}=v *$ $=v_{c}^{*}=\mathrm{V}^{*}=\Omega^{*}=1, \mathcal{L}_{T}^{*}=0.5$ and $\mathcal{L}_{\rho}^{*}=0.0$.

From figure (4) we observe that the growth rate is minimum for non thermally conducting medium while the higher values of thermal conductivity increased the instability growth rate. In other words we can say that the thermal conductivity has destabilizing influence on the system. 


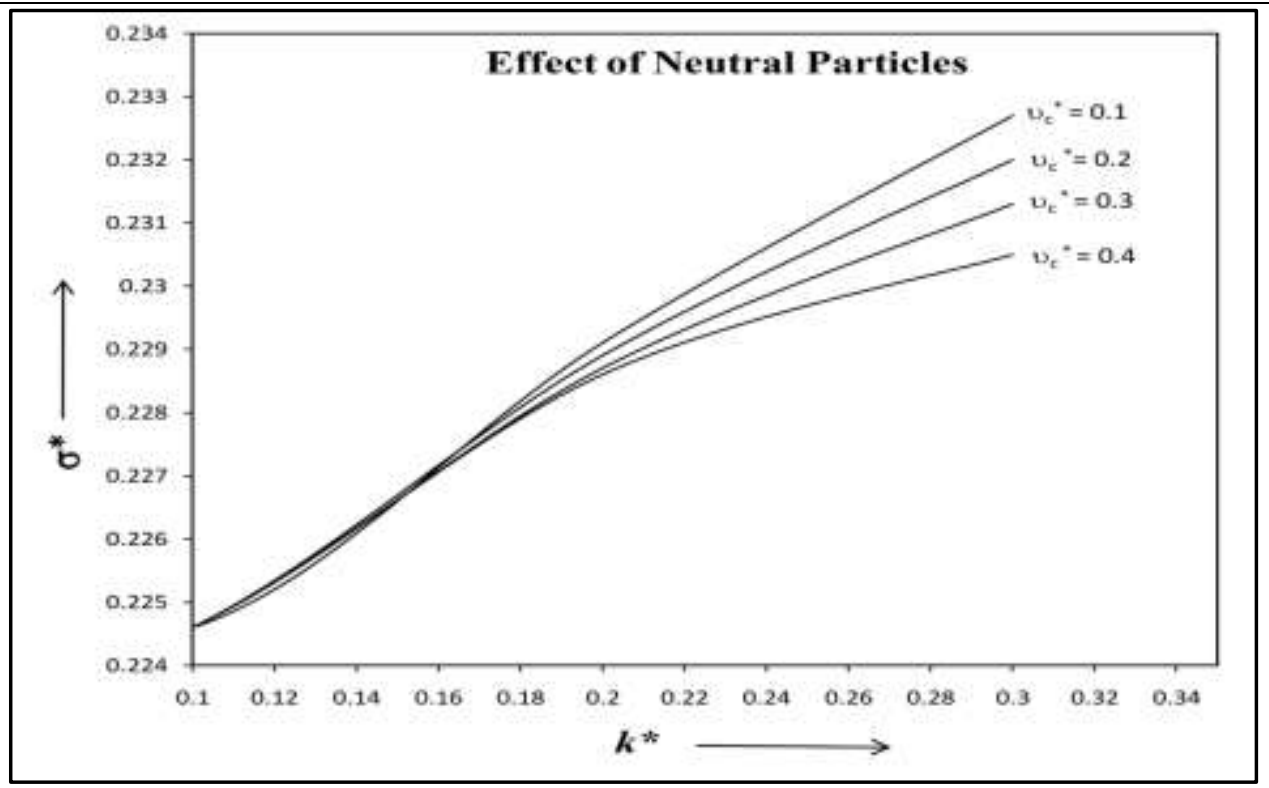

The growth rate is plotted against the non-dimensional wave number $\mathrm{k}^{*}$ with variation in the normalized neutral particle effect $v_{c}^{*}=0.1,0.2,0.3,0.4$ and the value of other parameter are fixed $\eta^{*}=$ $\nu^{*}=\mathrm{V}^{*}=\Omega^{*}=\lambda^{*}=1, \mathcal{L}_{T}^{*}=0.0$ and $\mathcal{L}_{\rho}^{*}=0.5$.

From the curves, we can analyze that increasing values of collision frequency decreases the growth rate of the system. In other words we can say that the role of neutral particles is to stabilize the plasma system.

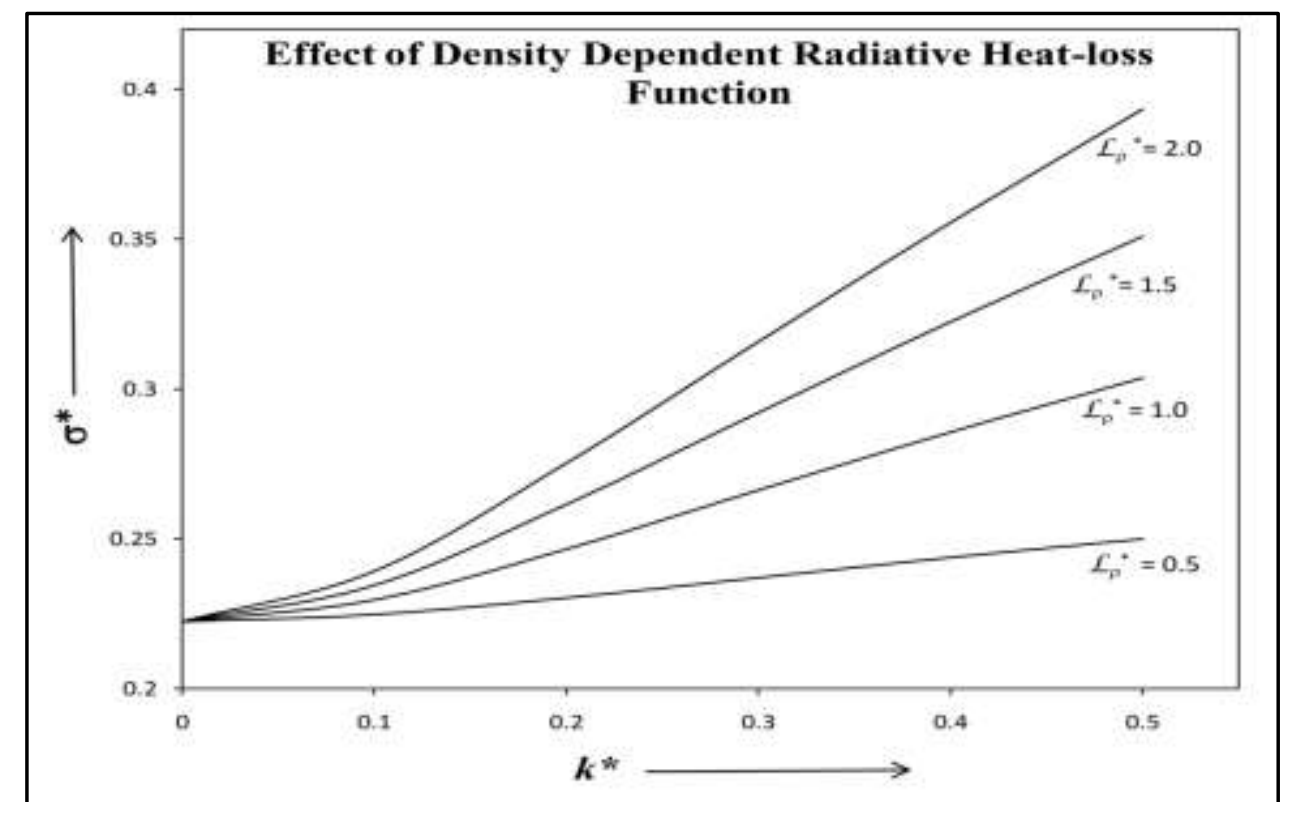

The growth rate is plotted against the non-dimensional wave number $\mathrm{k}^{*}$ with variation in the normalized density dependent heat-loss function $\mathcal{L}_{\rho}^{*}=0.5,1.0,1.5,2.0$. and the value of other parameter are fixed $\mathrm{V}^{*}=\eta^{*}=\Omega^{*}=\lambda^{*}=v^{*}=v_{c}^{*}=1$ and $\mathcal{L}_{T}^{*}=0.0$.

From figure (6) we analyze that the density dependent heat-loss function plays a same role as thermal conductivity and electrical resistivity play to destabilize the system. It means that the increasing values of density dependent heat-loss function increases the growth rate of instability.

\subsubsection{Axis of rotation perpendicular to the magnetic field}

We now analyze the wave propagation in transverse direction of external magnetic field considering the rotation of the magnetic field, we put $\Omega_{x}=\Omega$ and $\Omega_{z}=0$, the dispersion relation (46) reduces to

$$
-R_{1}\left(R_{1}^{2}+4 \Omega^{2}\right)\left[\sigma R_{1}+\sigma \frac{V^{2} k^{2}}{d}+\Omega_{T}^{2}\right]=0 .
$$


Equation (54) represents the dispersion relation for rotating, self-gravitating, viscous, partially ionized plasma incorporating the electrical resistivity, Hall current, thermal conductivity and radiation in transverse mode of propagation with axis of rotation perpendicular to the magnetic field. Equation (54) has three independent factors, each representing a different mode of propagation. The first factor of equation (54) equating to zero, gives

$$
\sigma^{2}+\sigma F+\Omega_{\mathrm{v}} v_{c}=0 \text {. }
$$

Equation (55) is identical with equation (27) and represents a viscous type of damped stable mode modified by the effects of viscosity collision frequency. The second factor of equation (54) equating to zero, gives

$$
\sigma^{4}+\sigma^{3} 2 F+\sigma^{2}\left[2 v_{\mathrm{c}} \Omega_{\mathrm{v}}+F^{2}+4 \Omega^{2}\right]+\sigma v_{\mathrm{c}}\left[2 \Omega_{\mathrm{v}} F+8 \Omega^{2}\right]+v_{c}^{2}\left(\Omega_{\mathrm{v}}^{2}+4 \Omega^{2}\right)=0 .
$$

This dispersion relation (56) is independent of finite electrical resistivity, self-gravitation, radiative heat-loss function, thermal conductivity, and magnetic field. This equation (56) represents a purely stable damped system due to the combined damping effects of rotation, collision frequency and viscosity of the medium. The third factor of equation (54) equating to zero gives.

$$
\begin{aligned}
& \sigma^{5}+\sigma^{4}\left[\Omega_{m}+v_{c}+v_{c} B+\Omega_{\mathrm{v}}+\beta\right]+\sigma^{3}\left[\Omega_{m}\left(v_{c}+v_{c} B+\Omega_{\mathrm{v}}+\beta\right)+\left(v_{c}+v_{c} B+\Omega_{\mathrm{v}}\right) \beta+\Omega_{\mathrm{v}} v_{c}+\right. \\
& V 2 k 2+\Omega j 2+\sigma 2 \Omega m \Omega j 2+\nu c+v c B+\Omega \mathrm{v} \beta+\Omega \mathrm{vvc} \beta+\Omega m+k 2 \mathrm{v} 2 \beta+\nu c+\Omega / 2+\nu c j 2+\sigma \Omega m \Omega \mathrm{v} v c \beta+\Omega \\
& \mathrm{v} \Omega j 2+\Omega / 2+\mathrm{v} 2 k 2 \beta \nu c+\nu c \Omega / 2+\nu c \Omega m \Omega / 2=0 .
\end{aligned}
$$

Equation (57) represents the dispersion relation for transverse wave propagating through finite homogeneous, self-gravitating, viscous magnetized partially ionized plasma having finite electrical resistivity, rotation, radiative effects, and thermal conductivity with the effect of neutral particles.

In the absence of collision frequency between two components of plasma, viscosity and electrical resistivity i.e. $v_{c}=0, v=0$ and $\eta=0$. with assumption that the fluid is non-magnetized $(H=0)$, the dispersion relation (57) reduces to as

$$
\sigma^{3}+\beta \sigma^{2}+\sigma \Omega_{J}^{2}+\Omega_{I}^{2}=0 .
$$

Equation (58) represents a dispersion relation for infinite homogeneous, non-magnetized, selfgravitating, thermally conducting plasma with radiative heat-loss effects. The condition of instability for such case is obtained from the constant term of equation (58), is given as

$$
k^{2}(\gamma-1)\left(\mathcal{L}_{T} T-\mathcal{L}_{\rho} \rho+\frac{\lambda k^{2} T}{\rho}\right)-4 \pi G \rho(\gamma-1)\left(\frac{\mathcal{L}_{T} T \rho}{p}+\frac{\lambda k^{2} T}{p}\right)<0 .
$$

This condition is same as condition (30) for longitudinal mode propagation. If fluid expressed by equation (57) is assumed magnetized i.e. $\mathrm{H} \neq 0$ then the dispersion relation becomes for such case as

$$
\sigma^{3}+\beta \sigma^{2}+\sigma\left(V^{2} k^{2}+\Omega_{J}^{2}\right)+\mathrm{V}^{2} k^{2} \beta+\Omega_{I}^{2}=0 .
$$

This is the dispersion relation for infinite homogeneous magnetized, self-gravitating, thermally conducting, and radiative effect. Condition of instability for this case is given as

$$
\left(V^{2} k^{2} \beta+k^{2} \alpha-4 \pi G \rho \beta\right)<0 .
$$

This is the modified condition of radiative instability due to the effect of magnetic field. This condition of instability (61) is identical to that of obtained by Aggrawal and Talwar [32], in the transverse mode of propagation. The effect of magnetic field comes through the term $V^{2} k^{2} \beta$ of magnetic field, there is an upward shift in the instability threshold i.e. the magnetic field decreases the value of critical wave number. Thus, we conclude that the magnetic field stabilizes the medium for transverse propagation.

\section{Conclusion}

In the Present analysis, we have studied the problem of an infinite homogeneous self-gravitating viscous and magnetized partially ionized fluid incorporating thermal conductivity, electrical resistivity, Hall current, rotation and radiative heat-loss functions. The general dispersion relation is obtained using linearized perturbation of the problem, which is modified due to the presence of these parameters. This general dispersion relation is reduced for longitudinal and transverse modes of propagation. We find that the Jeans condition remains valid but the expression of the critical Jeans wave number is modified due to presence of thermal conductivity and radiative heat-loss function. It is found that the viscosity parameter maintains the system stability in both, longitudinal and transverse direction of propagation.

The discussion of dispersion relation for longitudinal propagation, reveals that the viscosity, collision of neutrals with ionized gases, electrical resistivity, and Hall current modify the Alfven mode. The effect of the collision with neutrals does not affect the condition of instability of the considered fluid. It is also found that the effect of rotation does not contribute in the condition of radiative instability in both the direction parallel and perpendicular to the magnetic field.

In the transverse mode of propagation, we find that for an infinitely electrical conducting medium the condition of radiative instability is modified due to the presence of magnetic field and it shows a stabilizing influence. It is obtained that the gravitating thermal mode is affected by thermal conductivity and radiative heat- 
loss functions. We also found the condition of instability and the expression of critical Jeans wave number both are modified due to the presence of thermal conductivity, magnetic field, radiative heat-loss function and rotation. The effect of rotation in the condition of instability is observed only when the fluid is in-viscid and the axis of rotation is parallel to magnetic field. From the curves it is observed that thermal conductivity, electrical resistivity and density dependent heat-loss function have destabilizing influence on the instability of the fluid. It is also observed that the contribution of rotation and collision frequency it to reduce the growth rate and stabilize the system.

\section{References}

[1] J. H. Jeans, "The Stability of Spherical Nebula": Phil Trans. Ray. Soc. London A199, 1902, 1-53

[2] S. Chandrashekhar "Hydrodynamics and Hydromagnetic Stability": (Clarendon Press, Oxford, 1961).

[3] W. D. Langer, "The stability of interstellar clouds containing magnetic fields": Astrophysical Space Journal 225, 1978,95

[4] L. Mestel and L. Spitzer "Star Formation In Magnetic Dust Clouds": Mon. Not. Roy. Astron. Soc., 116, $1965,503$.

[5] L. Mestel "Problems of Star Formation I, II": Quart. J. Roy. Astron. Soc., 6, 1965, 161.

[6] L. Spitzer Physics of Fully Ionized Gases, (New-York inter science, 1962).

[7] L. Spitzer Diffuse Matter in Space, (New-York inter science, 1968).

[8] P. D. Ariel, "The character of equilibrium of an in-viscid, infinitely conducting fluid of variable density in the presence of a horizontal magnetic field with Hall-current": J. Plasma Phys. 4, 1970, 523-530.

[9] G. Bhowmick, "Rayleigh Taylor instability of a viscous Hall plasma with magnetic field": J. Plasma Phys. 7, $1972,117$.

[10] A. Ali and P. K. Bhatia "Magnetic resistivity and Hall currents effects on the stability of a self-gravitating plasma of varying density in variable magnetic field": Asrtophys. Space Sci. 201, 1993, 15-27.

[11] S. S. Kumar “On Gravitational Instability III”: Pub. Astron Soc .Japan. 13, 1961, 121-124.

[12] R. K. Chhajlani and D. S. Vaghela "Magnetogravitational stability of self-gravitating plasma with thermal conduction and finite Larmor radius through porous medium": Astrophys. Space Sci., 134, 1987, 301-315.

[13] M. K. Vyas, and R. K. Chhajlani "Gravitational instability of a thermally-conducting plasma flowing through a porous medium in the presence of suspended particles": Astrophys. Space Sci., 149, 1988, 323.

[14] R. C. Sharma and T. Chand, "Gravitational Instability for some Astrophysical Systems": Astrophys. Space Sci., 183(2), 1990, 215.

[15] A. Khan and P. K. Bhatia "Stability of two superposed viscoelastic fluids in a horizontal magnetic field": Indian J. Pure Appl. Math., 32(1), 2001, 99.

[16] P. K. Bhatia and R. P. S. Chhonkar: "Larmor radius effects on the instability of a rotating layer of a self-gravitating plasma": Astrophys. Space Sci. 115, 1985, 327-344.

[17] J. A. S Lima, R. Silva and J. Santos "Jeans Gravitational Instability and Non-Extensive Kinetic Theory": Astronomy and Astrophysics, 396, 2002, 309-314

[18] A. Khan and S. Shaikh "Instability of Thermally Conducting Self Gravitating System": J. Mod. Phys., 1, $2010,77-82$.

[19] R. K. Pensia, V. Kumar and V. Prajapat "Role of Magnetic Field in Contraction and Fragmentation of Interstellar Clouds": Ultra scientist, 22(2), 2010, 253-258.

[20] H. Stiele, H. Lesch, and F. Heitsch "Thermal instability in a weakly ionized plasma": Mon. Not. R. Astron. Soc. 372, $2006,862$.

[21] S. Inutsuka, H. Koyama, and T. Inoue, "The Role of Thermal Instability in Interstellar Medium": AIP conf. Proc., 784, 2005, 318.

[22] K. Menou, A. S. Balbus, and C. H. Spruit "Local Axisymmetric Diffusive Stability of Weakly Magnetized, Differentially Rotating, Stratified Fluids": Astrophys. J., 607, 2004, 564.

[23] W. Kim and R. Narayan "Turbulent Mixing in Clusters of Galaxies": Astrophys. J., 596, $2003,889$.

[24] M. Shadmehri and S. Dib "Magneto-thermal condensation modes including the effects of charged dust particles": Mon. Not. R. Astron. Soc., 395, 2009, 985.

[25] T. Fukue, and H. Kamaya "Small Structures via Thermal Instability of Partially Ionized Plasma I. Condensation Mode": Astrophys. J., 669, 2007, 363

[26] S. Shaikh, A. Khan and P. K. Bhatia "Stability of thermally conducting plasma in a variable magnetic field": Astrophys. Space Sci., 312, 2007, 35-40.

[27] S. Shaikh, A. Khan and P. K. Bhatia "Jeans gravitational instability of a thermally conducting plasma": Physics Lett. A 372, 2008, 1451 .

[28] B. K. Dangarh, R. K. Pensia, V. Shrivastava \& V. Prajapat “Analysis of Jeans Instability of Partially-Ionized Molecular Cloud under Influence of Radiative Effect and Electron Inertia": Adv. Studies Theor. Phys. 5(16), 2011, 755-764.

[29] S. Kaothekar, \& R. K. Chhajlani, "Gravitational instability of radiative plasma with finite Larmor radius corrections": Journal of Physics: conference series 365, 2012, 012044 .

[30] M. P. Bora, \& S. P. Talwar, "Magnetothermal Instability with Generalized Ohm's Law”: Phys. Fluids B5 (3), $1993,950$.

[31] R. K. Chhajlani and A. K. Parihar, "Stability of Self-Gravitating Magnetized Hall Plasma with Electrical and Thermal Conductivity through Porous Medium": Contrib. Plasma Phys. 33(3), 1993, 227-234.

[32] M. Aggarwal and S. P. Talwar, "Magneto-thermal instability in a rotating gravitating Fluid": Mon. Not. Roy. Astron. Soc. 146, 1969,235 